\title{
Differential binding of hyaluronan on the surface of tissue-specific endothelial cell lines
}

\author{
Karol Szczepanek ${ }^{1}$, Claudine Kieda ${ }^{2}$ and Joanna Cichy ${ }^{1 凶}$ \\ ${ }^{1}$ Department of Immunology, Faculty of Biochemistry, Biophysics and Biotechnology, Jagiellonian University, \\ Kraków, Poland; ${ }^{2}$ Centre de Biophysique Moléculaire, Glycobiologie, CNRS, Orléans, France
}

Received: 23 October, 2007; revised: 03 December, 2007; accepted: 25 January, 2008

available on-line: 30 January, 2008

\begin{abstract}
Tissue-specific heterogeneity of endothelial cells, both structural and functional, plays a crucial role in physiologic as well as pathologic processes, including inflammation, autoimmune diseases and tumor metastasis. This heterogeneity primarily results from the differential expression of adhesion molecules that are involved in the interactions between endothelium and circulating immune cells or disseminating tumor cells. Among these molecules present on endothelial cells is hyaluronan (HA), a glycosaminoglycan that contributes to primary (rolling) interactions through binding to its main receptor CD44 expressed on leukocytes and tumor cells. While the regulation of CD44 expression and function on either leukocytes or tumor cells has been well characterized, much less is known about the ability of endothelial cells to express HA on their surface. Therefore, in these studies we analyzed HA levels on tissue-specific endothelium. We used endothelial cell lines of different origin, including lung, skin, gut and lymph nodes that had been established previously as model lines to study interactions between the endothelium and leukocytes/tumor cells. Our results indicate that $\mathrm{HA}$ is accumulated on the surface of all endothelial cells examined. Moreover, retention of endogenous HA differs between the lines and may depend on their tissue origin. Analysis of binding of exogenous HA reveals the presence of specific HA binding sites on all endothelial cell lines tested. However, the retention of endogenous HA and the binding of exogenous $\mathrm{HA}$ is mediated through a CD44-independent mechanism.
\end{abstract}

Keywords: hyaluronan, CD44, inflammation, cell trafficking

\section{INTRODUCTION}

Hyaluronan (HA), a prevalent component of the extracellular and pericellular matrixes, is a nonsulfated glycosaminoglycan (GAG) ubiquitously expressed by various cell types in almost all animal tissues (Laurent \& Fraser, 1992). It has been demonstrated to play a key role in several biological processes including embryonic development, wound healing, tumor growth, and angiogenesis, mainly by providing a provisional matrix for supporting cellular migration and adherence (Laurent \& Fraser, 1992; Knudson, 1996; Camenisch \& McDonald, 2000; Lee \& Spicer, 2000). Tethering of hyaluronan to the cell surface is mediated by HA-binding proteins, such as CD44 and RHAMM (receptor for HA-mediated motility) of which CD44 is considered as a major HA cell surface receptor (Day \& Prestwich, 2002; Cichy \& Pure, 2003; Ponta et al., 2003). Glycoprotein CD44 is encoded by a single gene, but is expressed as multiple isoforms ranging in molecular mass from 80 to $250 \mathrm{kDa}$. The heterogeneity of CD44 mainly results from alternative splicing of variable exons as well as differential posttranslational modifications including glycosylation and the attachment of glycosaminoglycans (GAGs) (Cichy \& Pure, 2003; Ponta et al., 2003). Although CD44 is broadly expressed, including fibroblasts, keratinocytes, epithelial cells and leuko-

\footnotetext{
${ }^{\square}$ Corresponding author: Joanna Cichy, Department of Immunology, Faculty of Biochemistry, Biophysics and Biotechnology, Jagiellonian University, Kraków, Poland; tel.: (48) 12664 6135; fax: (48) 12664 6904; e-mail: cichy@mol.uj.edu.pl Abbreviations: $\mathrm{Ab}$, antibody; bHABP, biotinylated HA-binding protein; EC, endothelial cells; ECL, enhanced chemiluminescence; GAG, glycosaminoglycan; mAb, monoclonal antibody; HA, hyaluronan; RHAMM, receptor for HA-mediated motility; HRP, horseradish peroxidase; LPS, lipopolisaccharide; PBS, phosphate-buffered saline.
} 
cytes, in primary cells it usually does not exhibit a capacity to bind HA and requires activation to interact with its ligand. Notably, generation of an active, HA-binding form of this receptor is responsible for most of CD44's biological functions, such as myeloand leukopoiesis, leukocyte extravasation and activation (Cichy \& Pure, 2000; 2003; Ponta et al., 2003).

There is an emerging evidence that HA associated with vessel wall contributes to targeting lymphocytes to inflammatory sites (DeGrendele et al., 1997; Mohamadzadeh et al., 1998; Siegelman et al., 1999; 2000). Recognition of vascular endothelium by immune cells and subsequent migration of these cells through the vessel wall is mediated by a multistep process that primarily involves: i/ selectins and their carbohydrate ligands, ii/ chemoatractants and their receptors, and iii/ integrins interacting with proteins of the immunoglobulin superfamily (Butcher \& Picker, 1996). In addition, HA on endothelial cells can initiate contact and mediate rolling of circulating cells bearing active form of CD44 (DeGrendele et al., 1997; Siegelman et al., 1999; 2000). Interestingly, the CD44-HA interactions appear to be of particular significance in chronic inflammation and autoimmune diseases, including systemic lupus erythematosus and arthropathies where expression of CD44-dependent primary adhesion strongly correlates with concurrent symptomatic disease (Estess et al., 1998). The involvement of the CD44-HA complex in promoting chronic inflammatory conditions is also supported by findings showing that administration of anti-CD44 $\mathrm{Ab}$ that specifically blocks the CD44-HA interactions results in reduction of inflammatory symptoms such as $\mathrm{T}$ cell infiltrates and tissue edema in proteoglycan-induced arthritis, experimental allergic encephalomyelitis and insulin-dependent diabetes mellitus (Mikecz et al., 1995; 1999; Brocke et al., 1999; Weiss et al., 2000). In addition, CD44 and/or HA expressed on endothelial cells are well positioned to serve as an anchor for cancer cells disseminating through the blood stream. Indeed, CD44-mediated HA binding has been implicated in the adhesion of a leukemic cell line to vascular endothelial cells (Maiti et al., 1998).

Since the HA-mediated interactions between endothelium and leukocytes or disseminating tumor cells play an important role in extravasation of circulating cells, there is a great need to determine the potential of endothelial cells to express HA. On the other hand, endothelial cell lines showing heterogeneity in their HA surface expression may provide a potential tool for identifying mechanisms underlying HA-mediated migration across vascular endothelium. Bearing this in mind, in this work we characterized immortalized human endothelial cells for HA deposition on the cell surface. Our results indicate that levels of endogenously produced HA differ between cells of different origin. Moreover, we demonstrate that CD44 does not provide a docking site for HA on the surface of the endothelial cells examined.

\section{MATERIALS AND METHODS}

Reagents and antibodies. Biotinylated bovine hyaluronic acid-binding protein (bHABP) was purchased from US Biological (Massachusetts, MA, USA). Phycoerythrin (PE)-conjugated streptavidin was obtained from BD Pharmingen (San Diego, CA, USA). The following anti-human CD44 mAbs were used: Hermes III, fluorescein (FITC)-labeled C-26 (BD Pharmingen) and 5F12 that blocks HA binding (a generous gift of Dr. B. F. Haynes, Duke University Medical Center, Durham, NC, USA and Dr. E. Pure, Wistar Institute, Philadelphia, PA, USA). Purified hyaluronan from rooster comb was purchased from Sigma. Purified high molecular weight hyaluronan from human umbilical cord (HMW HA) was obtained from ICN Biomedicals (Costa Mesa, CA, USA). FITC-conjugated rooster comb HA (FITCHA) was prepared as described (de Belder \& Wik, 1975). FITC-conjugated anti-human CD3 antibody was purchased from BD Pharmingen. Anti-FITC Fab fragments of sheep IgG conjugated to horseradish peroxidase (HRP) were obtained from Roche Diagnostics (Mannheim, Germany). Hyaluronate lyase from Streptomyces hyalurolyticus was purchased from Sigma-Aldrich (Saint-Louis, MO, USA).

Cell culture. HLMEC - human lung microvascular endothelial cells derived from immortalized HS888Lu cells from normal lung tissue of a patient with osteosarcoma metastatic to the lung; HPLNEC. B3 - human peripheral lymph node endothelial cells taken from a cervical lymph node of a patient with Hodgkin's lymphoma; HSKMEC.1 - human skin microvascular endothelial cells from normal skin; HIMEC.1 - human intestine microvascular endothelial cells from normal intestine, and HAPEC.S1 - human appendix endothelial cells isolated from a biopsy of a patient with appendicitis were established as immortalized human endothelial cell lines as previously described (Bizouarne et al., 1993; Kieda et al., 2002). Human lung squamous carcinoma cell line (HTB58) and human breast adenocarcinoma (MDA-MB-231) were obtained from the American Type Culture Collection (Rockville, MD, USA). Cells were cultured in Dulbecco's Modified Eagle Medium (D-MEM) with high glucose (4.5 g/l D-glucose) supplemented with $50 \mu \mathrm{g} / \mathrm{ml}$ gentamycin and $2 \%$ heat-inactivated FBS (endothelial cells) or 10\% FBS (HTB58, MDA-MB231) (all reagents from Invitrogen, UK). Cells were plated, allowed to grow to confluence and used for experiments. 
Flow cytometry. Cells were harvested using 5 mM EDTA and stained as follows. The level of HA associated with the cell surface (endogenous HA) was determined after exposure of cells to bHABP followed by incubation with PE-conjugated streptavidin. Exogenous (soluble) HA binding was assayed using saturating amounts of FITC-conjugated HA. Specificity of FITC-HA binding to the cell surface was determined by preincubation with an excess of unlabeled high molecular weight HA (HMW HA) for 20 min followed by labeling with FITCHA. CD44-mediated HA binding was assayed using HA-FITC. Negative reactivity was based on staining with HA-FITC in the presence of blocking anti-CD44 $\mathrm{mAb} 5 \mathrm{~F} 12$. To detect CD44, cells were stained with FITC-conjugated mouse anti-human CD44 antibody (C-26) or FITC-conjugated mouse anti-human CD3 antibody as a negative control. All the results were obtained using FACScan and CellQuest software (BD Biosciences, San Jose, CA, USA).

Western blot analysis. Cells were lysed in PBS containing $1 \%$ Nonidet P-40, $0.1 \%$ sodium deoxycholate, and protease inhibitors $(1 \mathrm{mg} / \mathrm{ml}$ aprotinin, $100 \mu \mathrm{g} / \mathrm{ml}$ Pefabloc SC, $100 \mu \mathrm{g} / \mathrm{ml}$ leupeptin, and $100 \mu \mathrm{g} / \mathrm{ml}$ trypsin inhibitor). Cell lysates were normalized based on protein concentrations as determined using the BCA kit (Sigma-Aldrich) and equal amounts of protein were subjected to immunoprecipitation with anti-CD44 mAb (Hermes III) conjugated to CNBr-activated Sepharose (Amersham Pharmacia). Immune complexes were washed once with lysis buffer followed by three washes with PBS, and resolved on SDS/8\% PAGE under nonreducing conditions. CD44 was visualized by enhanced chemiluminescence (ECL) (Pierce, Rockford, IL, USA) after electrotransfer to a PVDF membrane (Amersham Pharmacia Biotech) and incubation with FITC-conjugated mouse anti-CD44 antibodies (C-26) and anti-FITC Fab fragments of sheep IgG conjugated to HRP.

\section{RESULTS}

Tissue-specific endothelial cell lines are heterogenous with respect to levels of $\mathrm{HA}$ associated with the cell surface

We employed immortalized human endothelial cell lines originated from different tissues/organs as a model to determine whether HA is expressed on endothelial cells in a tissue-specific manner. The cells were isolated both from lymphatic organs (peripheral lymph nodes, appendix) and nonlymphatic sites (skin, lung and intestine). For each type of endothelium different clones were obtained following immortalization and tested for endothelial features, such as the presence of von Willebrand factor, Eand P-selectin, CD34 and MAdCAM-1 (Kieda et al., 2002). Clones that most-closely resembled tissuespecific endothelium were chosen for these studies (Bielawska-Pohl et al., 2005).

Surface levels of endogenously produced HA were assessed by flow cytometry using HAbinding protein (bHABP). As shown in Fig. 1, all endothelial cells (EC) expressed this GAG on their surface. However, EC isolated from lung (HLMEC) expressed the highest levels of HA followed by intestine (HIMEC.1), lymph-node (HPLNEC.B3), skin (HSKMEC) and appendix-derived HAPEC.S1. Notably, the levels of HA associated with the surface of lung- (HLMEC) and intestine-derived endothelial cells (HIMEC.1) were higher compared with tumor cells originating from lung and breast epithelial cells (HTB58 and MDA-MB-231, respectively) (Fig. 1.), which had been previously shown to express significant amounts of HA (Yu et al., 1997; Cichy \& Pure, 2000; 2004). These results suggest that, similar to other adhesion molecules (Butcher et al., 1999), the amount of HA retained on endothelium may depend on its tissue origin.

\section{Exogenous HA is bound by EC}

Endothelium lining blood vessels is characterized by a thick surface coat known as glycocalix that is mainly composed of glycoproteins and glycosaminoglycans (Baldwin \& Thurston, 2001). Since all endothelial cell lines tested displayed significant levels of surface HA, we next examined whether all HA-binding sites are saturated with endogenous HA and whether these cells are still capable of binding additional exogenous HA. Cells were incubated with FITC-labeled exogenous $\mathrm{HA}$ in the presence or absence of an excess of unlabeled high molecular weight HA (HMW HA) that was used as a competitive inhibitor. All lines tested except appendix-derived HAPEC.S1 exhibited significant affinity for exogenous HA (Fig. 2), indicating the presence of HA-binding sites on their surface.

There is accumulating evidence that surface HA binding can be attributed to the presence of CD44 in its active form (Cichy \& Pure, 2000; 2004; Cichy et al., 2002). In order to determine whether CD44 mediates HA binding on the endothelial cells, we used anti-CD44 antibody 5F12 which blocks the interaction between $\mathrm{HA}$ and CD44 (Cichy \& Pure, 2000). As demonstrated in Fig. 2, $5 \mathrm{~F} 12 \mathrm{mAb}$ did not significantly inhibit the binding of FITC-labeled HA to the surface of EC lines. In contrast, treatment with $5 \mathrm{~F} 12 \mathrm{mAb}$ abrogated soluble HA binding to HTB58 and MDA-MB-231 
A
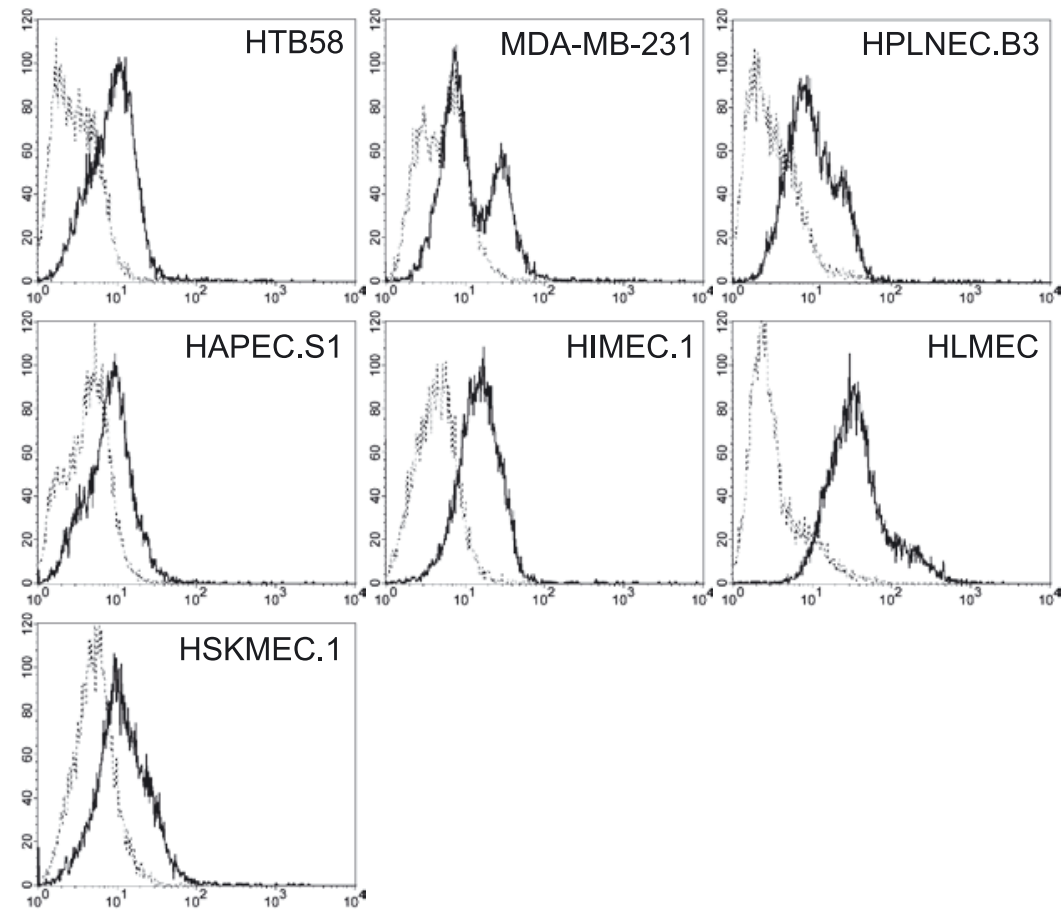

Fluorescence intensity

B

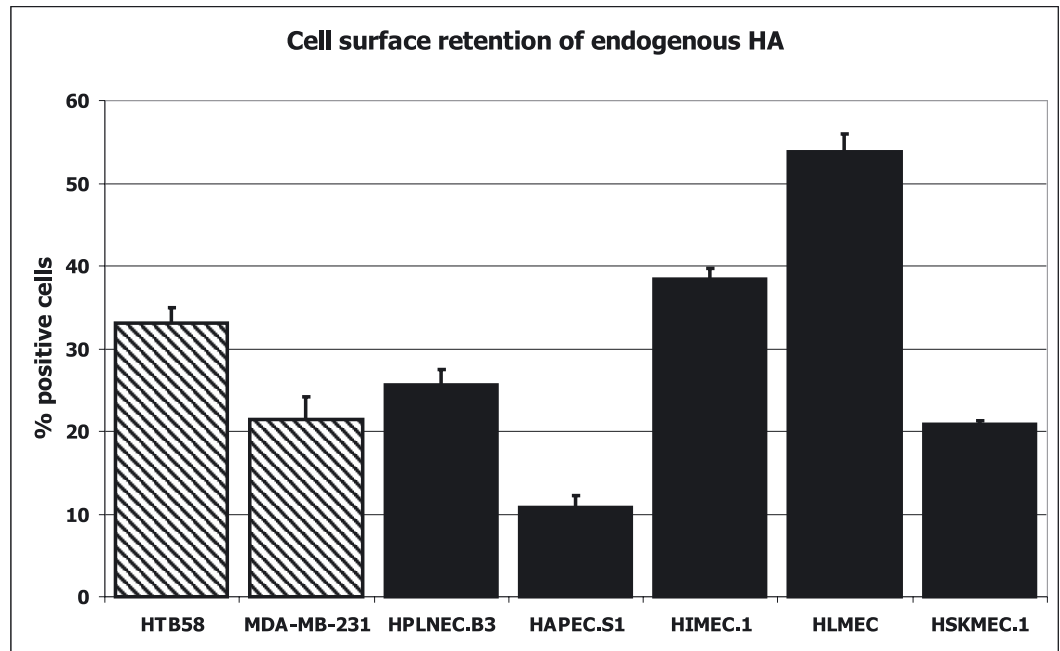

Figure 1. Basal levels of endogenous HA differ among endothelial cell lines.

Flow cytometric analysis of endogenous HA levels on endothelial cells isolated from different lymphatic (HPLNEC.B3 and HAPEC.S1) and nonlymphatic tissues (HIMEC.1, HLMEC and HSKMEC.1). The levels of HA associated with cell surface of nonendothelial cancer cells, HTB58 and MDA-MB-231, are shown as a control. Cells were labeled with biotinylated HA-binding protein (bHABP), followed by incubation with PE-conjugated streptavidin (solid lines) or PE-streptavidin only (dashed lines). (A) Mean fluorescence intensity from one representative experiment out of three independent experiments is shown. (B) Percentage of positive cells is shown as the mean \pm S.D. from three independent experiments. epithelial tumor cells that express functional CD44 (Cichy \& Pure, 2000). The effect of anti-CD44 mAb on HA binding in tumor cells was similar to the effect of unlabeled high molecular weight HA, both of which diminished FITC-HA staining to baseline levels (Fig. 2). Taken together, these data indicate that CD44 does not participate in HA binding on the surface of tissue-specific endothelial cell lines.

\section{EC do not express CD44}

Since CD44 needs activation to bind HA, the CD44-independent HA binding on endothelial cells could result from expression of functionally inactive CD44 or a lack of this receptor on the cell surface under the experimental conditions. As shown in Fig. 3, flow cytometry analysis did not reveal CD44 surface expression on any endothelial cell line examined. As expected, significant levels of CD44 were detected in HTB58 and MDA-MB-231 tumor cells, consistent with previous reports (Cichy \& Pure, 2000; Cichy et al., 2005). To determine whether endothelial cells are only deficient in surface CD44 or do not express CD44 at all, we performed CD44-specific immunoprecipitations followed by Western blot analysis. As demonstrated in Fig. 4, 


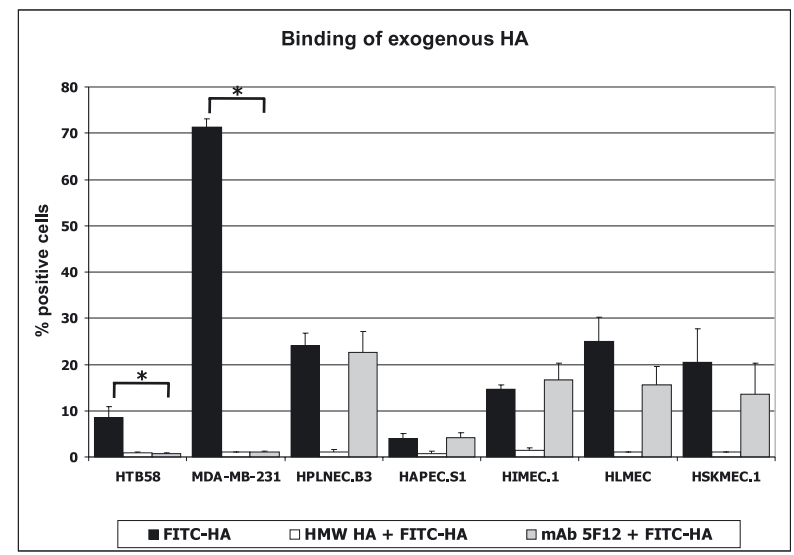

Figure 2. Tissue-specific endothelial cells bind soluble exogenous HA.

Cells were labeled with FITC-conjugated HA and analyzed by flow cytometry (black bars). Nonspecific HA binding was determined by incubation with FITC-HA in the presence of an excess of high molecular weight unlabeled HA (HMW HA) (white bars). Involvement of CD44 in soluble HA binding was determined by pretreatment of cells with blocking anti-CD44 mAb 5F12 followed by incubation with FITC-HA (grey bars). Percentage of positive cells is shown as the mean \pm S.D. from three independent experiments. The difference between HA binding in the presence or absence of blocking anti-CD44 mAb 5F12 was statistically significant (Student's $t$-test $P<0.05$ ) only for HTB58 and MDA-MB-231 cells (*).

a predominant $90 \mathrm{kDa}$ form and two additional species of CD44 with average molecular masses of $110 \mathrm{kDa}$ and $180 \mathrm{kDa}$ were immunoprecipitated from lysates of HTB58 cells and MDA-MB-231 tumor cells. However, CD44 could not be detected in lysates of any of the tested endothelial cells, in-

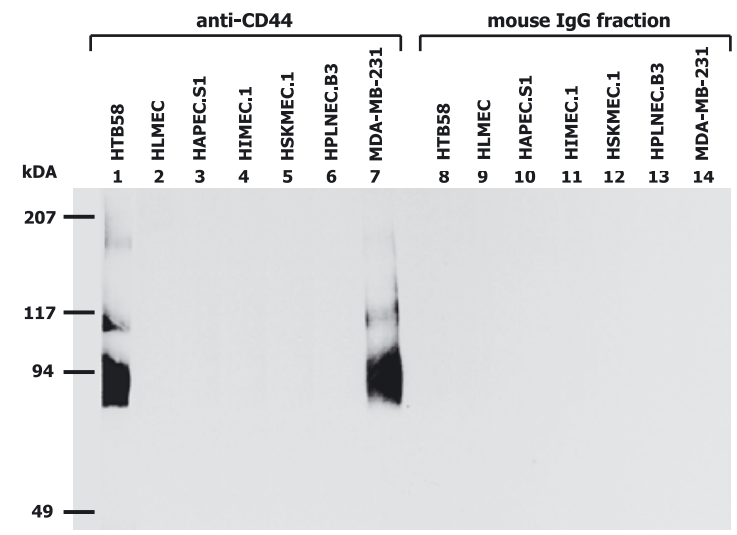

Figure 4. Western blot analysis confirms lack of CD44 expression in endothelial cells.

Indicated endothelial cells were lysed and half of the cell lysates were immunoprecipitated with Sepharose-conjugated anti-CD44 mAb Hermes III (lanes 1-7). To show specificity, the remaining half of the cell lysates was subjected to immunoprecipitation with total mouse IgG (lanes 8-14). All samples were separated on SDS/PAGE under nonreducing conditions, transferred to PVDF membrane, and incubated with mouse FITC-conjugated anti-human CD44 $\mathrm{mAb}$ followed by HRP-conjugated anti-FITC Ab. Reactivity was then detected by ECL. Molecular mass markers in $\mathrm{kDa}$ are indicated.

dicating that CD44 is unlikely to contribute to the HA-related heterogeneity among EC.

\section{DISCUSSION}

Although extracellular organization and function of $\mathrm{HA}$ in a number of solid tissue micro-

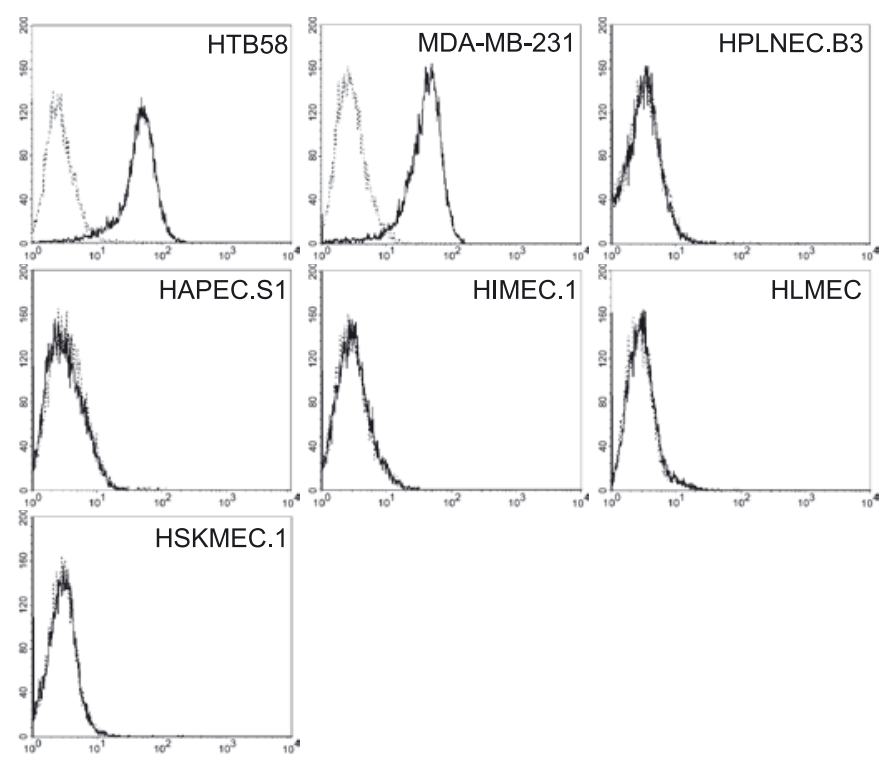

Fluorescence intensity
Figure 3. CD44 is not expressed on the surface of endothelial cells.

Cell surface levels of CD44 were analyzed by flow cytometry after staining with FITC-conjugated anti-CD44 mAb (solid line) and a negative control FITC-conjugated anti-CD3 (dashed line). Results are expressed as mean fluorescence intensity of one representative experiment out of three independent experiments. 
environments has been extensively examined, its deposition on the endothelium remains to be fully elucidated. HA has been reported to be associated with dermal microvasculature in skin of patients suffering from psoriasis (Tammi et al., 1994), indicating the presence of this GAG on the endothelium in vivo under conditions of chronic inflammation. Moreover, endothelial cell lines and primary cultures of endothelial cells can be induced by proinflammatory mediators to express surface HA. Interestingly, the up-regulation of HA levels mediated by TNF $\alpha$, IL1 $\beta$ and LPS appears to be restricted to small venular types of endothelium, consistent with a role of this type of endothelium in mediating leukocyte recruitment to inflammatory sites (Mohamadzadeh et al., 1998). In addition, arterial, microvessel and large vein-derived endothelial cells have been described to differ in their ability to bind exogenous HA (Lokeshwar \& Selzer, 2000). Taken together, these data support the notion that endothelial cells of different vascular origins show heterogeneity in HA-mediated functions.

The studies presented here suggest that the amount of HA anchored to the endothelial cells may also be dependent on the tissue of origin of these cells. This is important in view of the fact that endothelium-specific addressins, including MAdCAM-1, GlyCAM-1 and CD34 significantly contribute to region/organ specific homing of immune cells (Butcher et al., 1999). Therefore, the differential levels of HA on EC derived from various tissues suggest that HA-mediated binding of circulating cells to endothelium may occur in a tissue-specific manner. However, we can not exclude the possibility that cell immortalization and/or tissue culture conditions also contribute to the observed heterogeneity among the endothelial cells studied.

The overall lower levels of HA retained on endothelial cells isolated from lymphatic tissues (particularly from appendix), compared to EC derived from nonlymphatic sites such as lung and intestine suggest that endothelial HA is more likely to support cell trafficking to extralymphatic sites. These results are consistent with the findings that $\mathrm{T}$ cell CD44-mediated interactions do not contribute to $\mathrm{T}$ cell recruitment to lymphoid tissues in the physiological state but are rather involved in the trafficking of activated lymphocytes to the sites of inflammation or injury (Camp et al., 1993).

The EC heterogeneity with respect to surface HA levels could result from differences in the synthesis of this GAG, availability of functional HA-binding molecules on the cell surface, or a combination thereof. Since the different EC examined were capable of binding exogenous HA, the
HA-binding sites were not saturated in these cells, potentially allowing binding of higher amounts of endogenously produced HA. Therefore differential HA expression in tissue-specific EC could be at least partially responsible for the observed differences in HA surface levels among these cells. On the other hand, appendix-derived HAPEC.S1 endothelial cells that were found to contain the lowest amount of HA on the cell surface also displayed the lowest ability to bind soluble HA, indicating that HAPEC.S1 cells are deficient in HA-binding receptors. Taken together these results demonstrate that HA deposition on EC is dependent on both, HA synthesis and the availability of HA binding membrane molecules.

In order to characterize the HA-binding sites on tissue-specific endothelial cells, we focused on CD44, which is well known to mediate immobilization of HA on a variety of cells (Knudson et al., 1996; Cichy \& Pure, 2003). In addition, several reports have demonstrated CD44 expression on EC, including proliferating HUVEC and bovine aortic EC (Griffioen et al., 1997). However, our findings clearly indicate that CD44 is unlikely to serve as an anchor for HA on EC. Even though CD44 is considered a principal receptor for HA, other molecules may be involved in HA binding on endothelial cells since this GAG is well-known to interact with a large number of proteins collectively known as hyaladherins. These include RHAMM and several components of the cellular matrix such as aggrecan and versican (Day \& Prestwich, 2002). In addition, HA synthetases that are localized on the cytoplasmic surface of the cell membrane could potentially contribute to the retention of HA on cell surfaces (Camenisch \& McDonald, 2000). Notably, in agreement with our findings, RHAMM but not CD44 has been implicated in mediating soluble HA binding on arterial, microvessel and vein-derived human EC (Lokeshwar \& Selzer, 2000). Taken together these data indicate that, in contrast to other cells such as chondrocytes and epithelium that interact with HA in a CD44-dependent manner (Yu et al., 1997; Cichy \& Pure, 2000; Knudson et al., 2002), endothelial cells appear less likely to utilize this receptor for HA binding and concomitantly assembly of the pericellular matrix based on a hyaluronan scaffold. Another indirect evidence supports structural differences in the HA-based matrix associated with EC compared to the HA-based matrix associated with epithelial tumor cell lines. This evidence is related to various effects of the HAdegrading enzyme - a hyaluronidase on the disruption of the HA-cell interactions in these cells. Whereas pretreatment of HTB58 or MDA-MB-231 tumor cells with hyaluronidase resulted in block- 
ing of bPG staining, hyaluronidase was not effective in removing surface HA from endothelial cells (not shown).

Since the levels and organization of HA on the EC surface may function as important determinants of EC biology under physiologic and pathologic conditions, the characterized EC lines represent a convenient tool to study the rolling interactions between circulating blood-borne leukocytes or cancer cells with tissue-specific endothelium.

\section{Acknowledgements}

This work was supported by grant from EU 6th FP project SP6MTKD-CT-2006-042586.

\section{REFERENCES}

Baldwin AL, Thurston G (2001) Mechanics of endothelial cell architecture and vascular permeability. Crit Rev Biomed Eng 29: 247-278.

Bielawska-Pohl A, Crola C, Caignard A, Gaudin C, Dus D, Kieda C, Chouaib S (2005) Human NK cells lyse organspecific endothelial cells: analysis of adhesion and cytotoxic mechanisms. J Immunol 174: 5573-5582.

Bizouarne N, Denis V, Legrand A, Monsigny M, Kieda C (1993) A SV-40 immortalized murine endothelial cell line from peripheral lymph node high endothelium expresses a new alpha-L-fucose binding protein. Biol Cell 79: 209-218.

Brocke S, Piercy C, Steinman L, Weissman IL, Veromaa T (1999) Antibodies to CD44 and integrin alpha4, but not L-selectin, prevent central nervous system inflammation and experimental encephalomyelitis by blocking secondary leukocyte recruitment. Proc Natl Acad Sci USA 96: 6896-6901.

Butcher EC, Picker LJ (1996) Lymphocyte homing and homeostasis. Science 272: 60-66.

Butcher EC, Williams M, Youngman K, Rott L, Briskin M (1999) Lymphocyte trafficking and regional immunity. Adv Immunol 72: 209-253.

Camenisch TD, McDonald JA (2000) Hyaluronan: is bigger better? Am J Respir Cell Mol Biol 23: 431-433.

Camp RL, Scheynius A, Johansson C, Pure E (1993) CD44 is necessary for optimal contact allergic responses but is not required for normal leukocyte extravasation. J Exp Med 178: 497-507.

Cichy J, Pure E (2000) Oncostatin M and transforming growth factor-beta 1 induce post-translational modification and hyaluronan binding to CD44 in lung-derived epithelial tumor cells. J Biol Chem 275: 18061-18069.

Cichy J, Pure E (2003) The liberation of CD44. J Cell Biol 161: 839-843.

Cichy J, Pure E (2004) Cytokines regulate the affinity of soluble CD44 for hyaluronan. FEBS Lett 556: 69-74.

Cichy J, Bals R, Potempa J, Mani A, Pure E (2002) Proteinase-mediated release of epithelial cell-associated CD44. Extracellular CD44 complexes with components of cellular matrices. J Biol Chem 277: 44440-44447.

Cichy J, Kulig P, Pure E (2005) Regulation of the release and function of tumor cell-derived soluble CD44. Biochim Biophys Acta 1745: 59-64.

Day AJ, Prestwich GD (2002) Hyaluronan-binding proteins: tying up the giant. J Biol Chem 277: 4585-4588. de Belder AN, Wik KO (1975) Preparation and properties of fluorescein-labelled hyaluronate. Carbohydr Res 44: 251-257.

DeGrendele HC, Estess P, Siegelman MH (1997) Requirement for CD44 in activated T cell extravasation into an inflammatory site. Science 278: 672-675.

Estess P, DeGrendele HC, Pascual V, Siegelman MH (1998) Functional activation of lymphocyte CD44 in peripheral blood is a marker of autoimmune disease activity. J Clin Invest 102: 1173-1182.

Griffioen AW, Coenen MJ, Damen CA, Hellwig SM, van Weering DH, Vooys W, Blijham GH, Groenewegen G (1997) CD44 is involved in tumor angiogenesis; an activation antigen on human endothelial cells. Blood 90: 1150-1159.

Kieda C, Paprocka M, Krawczenko A, Zalecki P, Dupuis P, Monsigny M, Radzikowski C, Dus D (2002) New human microvascular endothelial cell lines with specific adhesion molecules phenotypes. Endothelium 9: 247-261.

Knudson W (1996) Tumor-associated hyaluronan. Providing an extracellular matrix that facilitates invasion. $\mathrm{Am}$ J Pathol 148: 1721-1726.

Knudson W, Aguiar DJ, Hua Q, Knudson CB (1996) CD44anchored hyaluronan-rich pericellular matrices: an ultrastructural and biochemical analysis. Exp Cell Res 228: 216-228.

Knudson W, Chow G, Knudson CB (2002) CD44-mediated uptake and degradation of hyaluronan. Matrix Biol 21: 15-23.

Laurent TC, Fraser JR (1992) Hyaluronan. FASEB J 6: $2397-$ 2404.

Lee JY, Spicer AP (2000) Hyaluronan: a multifunctional, megaDalton, stealth molecule. Curr Opin Cell Biol 12: 581-586.

Lokeshwar VB, Selzer MG (2000) Differences in hyaluronic acid-mediated functions and signaling in arterial, microvessel, and vein-derived human endothelial cells. $J$ Biol Chem 275: 27641-27649.

Maiti A, Maki G, Johnson P (1998) TNF-alpha induction of CD44-mediated leukocyte adhesion by sulfation. Science 282: 941-943.

Mikecz K, Brennan FR, Kim JH, Glant TT (1995) Anti-CD44 treatment abrogates tissue oedema and leukocyte infiltration in murine arthritis. Nat Med 1: 558-563.

Mikecz K, Dennis K, Shi M, Kim JH (1999) Modulation of hyaluronan receptor (CD44) function in vivo in a murine model of rheumatoid arthritis. Arthritis Rheum 42: 659-668.

Mohamadzadeh M, DeGrendele H, Arizpe H, Estess P, Siegelman M (1998) Proinflammatory stimuli regulate endothelial hyaluronan expression and CD44/HA-dependent primary adhesion. J Clin Invest 101: 97-108.

Ponta H, Sherman L, Herrlich PA (2003) CD44: from adhesion molecules to signalling regulators. Nat Rev Mol Cell Biol 4: 33-45.

Siegelman MH, DeGrendele HC, Estess P (1999) Activation and interaction of CD44 and hyaluronan in immunological systems. J Leukoc Biol 66: 315-321.

Siegelman MH, Stanescu D, Estess P (2000) The CD44-initiated pathway of T-cell extravasation uses VLA-4 but not LFA-1 for firm adhesion. J Clin Invest 105: 683-691.

Tammi R, Paukkonen P, Wang C, Horsmanheimo M, Tammi M (1994) Hyaluronan and CD44 in psoriatic skin. Intense staining for hyaluronan on dermal capillary loops and reduced expression of CD44 and hyaluronan in keratinocyte-leukocyte interfaces. Arch Dermatol Res 286: $21-29$. 
Weiss L, Slavin S, Reich S, Cohen P, Shuster S, Stern R, Kaganovsky E, Okon E, Rubinstein AM, Naor D (2000) Induction of resistance to diabetes in non-obese diabetic mice by targeting CD44 with a specific monoclonal antibody. Proc Natl Acad Sci USA 97: 285-290.
Yu Q, Toole BP, Stamenkovic I (1997) Induction of apoptosis of metastatic mammary carcinoma cells in vivo by disruption of tumor cell surface CD44 function. J Exp Med 186: 1985-1996. 